\title{
AvallaÇÃo dA FReqüênCIA DE DEPRESSÃO EM PACIENTES COM ENDOMETRIOSE E DOR PÉLVICA
}

\author{
Carolina lorençatto, Maria José Navarro Vieira, Cristina laguna B. Pinto, Carlos alberto Petta* \\ Trabalho realizado no Ambulatório de Endometriose do Departamento de Tocoginecologia \\ do Centro de Atenção Integral à Saúde da Mulher (CAISM/FCM/UNICAMP), Campinas, SP.
}

Resumo - Oвjetivo. Avaliar a freqüência de depressão em pacientes com endometriose e queixa atual de dor pélvica para determinar a necessidade ou não de atendimento psicológico.

Mítodos. Participaram deste estudo 50 mulheres com idade entre 24 e 48 anos, com diagnóstico de endometriose e queixa atual de dor pélvica. Para avaliar a prevalência da depressão, utilizou-se - Inventário de Depressão de Beck, validado em nosso meio.

Resultados. Identificou-se a presença de depressão em $92 \%$ das pacientes avaliadas, sendo $56 \%$ de intensidade moderada a grave.
Apesar de $66 \%$ das pacientes relatarem o uso de medicação, não foi observada correlaçäo significativa entre a presença de depressão com 0 uso de hormônios.

Conclusío. Os resultados demostraram uma alta prevalência de depressão, justificando-se, assim, a necessidade do atendimento psicológico a essas pacientes.

UnITERMOS: Endometriose. Dor pélvica. Depressão. Atendimento psicológico.

\section{INTRODUÇÃO}

A endometriose é uma ginecopatia que acomete mulheres em idade reprodutiva desde a puberdade até a menopausa. Consiste na presença de endométrio em locais fora do útero, sendo uma doença progressiva que pode provocar lesões no aparelho reprodutor, e como resultado disso, o aparecimento de manifestações dolorosas e infertilidade!.

Estima-se que a endometriose seja responsável por $40 \%$ das queixas de dor pélvica crônica e por $35 \%$ dos casos de infertilidade feminina. Aincidência média da doença é bastante discutida; acredita-se atualmente que atinge em torno de $15 \%$ na população feminina em idade reprodutiva ${ }^{1,2}$.

Considerada como doença enigmática, de etiopatogenia incerta e tratamento variável, a endometriose é referenciada como "doença da mulher moderna", sendo analisada pelo ponto de vista biopsicossocial. Atualmente, a mulher está propensa a uma menarca mais precoce, menor número de gestações (e cada vez mais tardias), o que implicaria em maior número de menstruações e, portanto, maior

*Correspondência:

Caixa Postal 6181 - Cep: 13083-970

Campinas - SP - Fone: (19) 32892856

Fax: (19) 32892440 exposição à menstruação retrógrada. Além disso, a videolaparoscopia, que é um método que se popularizou nos últimos anos, possibilitou um maior número de diagnósticos ${ }^{1,3}$.

O estresse e a ansiedade são vistos como fatores que poderiam contribuir para o desenvolvimento da endometriose, como também cronificar o processo etiológico'. Por isso, para alguns autores, a avaliação do perfil psicológico da paciente pode ser elemento de ajuda para o diagnóstico de endometriose. Sugere-se que essas mulheres apresentam certas características emocionais perceptíveis na prática clínica: elevado coeficiente intelectual, perfeccionismo, egocentrismo, ansiedade e estresse psíquico ${ }^{4}$.

Buscando encontrar um perfil psicológico associado à endometriose, Low et al. ${ }^{5}$ avaliaram 81 mulheres com dor pélvica, sendo 40 casos de endometriose e $4 \mathrm{I}$ de outras ginecopatias. As pacientes com endometriose apresentaram índices mais elevados de introversão e ansiedade quando comparado ao outro grupo.

Alguns traços de personalidade de pacientes com endometriose foram delineados através de um estudo exploratório conduzido por Abrão et al.'. São eles: ansiedade, auto-exigência, insegurança, mecanismos de defesa estruturados, alta capacidade de controle e comando, intolerância diante das falhas huma- nas ou frente a situações de frustrações, nãocontato com suas emoções e onipotência. Com isso, esses autores afirmam existir um perfil emocional característico em comum nas mulheres portadoras de endometriose.

Questiona-se paralelamente a relação entre o perfil psicológico e a intensidade da dor relatada pela paciente com endometriose e dor pélvica crônica, sugerindo a existência de uma desordem na personalidade devidoà falta de correlação entre a presença dos sintomase a extensão da doença ${ }^{3}$. O que torna a endometriose ainda uma doença enigmática é o fato de muitas mulheres com comprometimento pélvico aparente serem assintomáticas, e outras, com comprometimento pélvico mínimo, queixarem-se de dores fortes, entre outros sintomas. Esta é a principal razão para verificar se existem diferenças no perfil psicológico de determinadas pacientes que estão associadas ao aparecimento dos sintomas, principalmente da dor pélvica crônica.

Existem controvérsias nos estudos que avaliam a quantidade de dor relatada pela paciente eas características da endometriose, como quantidade, local de acometimento e tipo de lesão. Em um recente estudo internacional, os autores não encontraram relação nítida entre a dor referida e características morfológicas da endometriose, bem como com o local e estágio da doença ${ }^{6}$. 
Considera-se que a dor pélvica crônica possa causar prejuízos físicos, psíquicos e sociais, assim como qualquer doença crônica, pois restringe e modifica o convívio diário da paciente com suas rotinas até então estabelecidas. Além da endometriose, existem várias outras causas de origem física para explicar a presença de dor pélvica crônica. Porém, acredita-se que o fator psicológico pode estar presente de forma isolada ou concomitante em até $60 \%$ dos casos, sendo mais comum os sintomas de depressão e ansiedade ${ }^{7}$.

É possível afirmar que todas as experiências humanas, inclusive a dolorosa, envolvem componentes físicos e emocionais. As emoções mais comuns associadas à dor crônica são, em geral, depressão e ansiedade. O humor pode ficar comprometido interferindo na interpretação e no relato da dor ${ }^{8}$.

Adepressão representa um grupo heterogêneo de sintomas. Algumas das manifestações mais comum são: humor deprimido, diminuição acentuada no interesse e no prazer pelas atividades anteriormente satisfatórias, perda ou aumento significativo do peso, insônia ou hipersônia, agitação ou retardo psicomotor, fadiga, pensamentos de morte, entre outros. Dependendo da freqüência e intensidade desses sintomas, a depressão é classificada em leve, moderada ou grave, sendo que para 0 diagnóstico efetivo é necessário um período mínimo de duas semanas, nas quais predomina humor deprimido ou perda de interesse por quase todas as atividades. Devido a isso, observa-se comprometimento no funcionamento social, profissional e afetivo, que agrava-se de acordo com a intensidade dos sintomas s,9. $^{8,9}$

Observa-se que na população feminina, esseéum transtorno mental de grande importância médico-social. A prevalência é bastante discutida entre autores, mas de acordo com o Manual Diagnóstico e Estatístico de Transtornos Mentais (DSM-IV)' a prevalência-ponto do transtorno depressivo tem variado de $5 \%$ a $9 \%$ para as mulheres, não se relacionando à fatores como etnia, educação, rendimentos ou estado civil.

Ainda hoje, a depressão é uma doença pouco reconhecida, mal diagnosticada e mal tratada. Pode manifestar-se isoladamente ou associada a uma outra doença. Devido a diversidade de sintomas experienciados, é comum que pacientes depressivos procurem especia- listas de diversas áreas apresentando queixas somáticas relacionadas ao estado afetivo comprometido ${ }^{10}$.

O diagnóstico é basicamente clínico, tendo como parâmetros os critérios definidos pelo DSM-IV ${ }^{9}$ e/ou Classificação Internacional de Doenças (CID- IO)". Aentrevista clínicaéo recurso mais utilizado para realizar o diagnóstico, sendo possível identificar a presença e intensidade da depressão. As escalas de avaliação da depressão foram elaboradas para auxiliar na avaliação dos sintomas ena elaboração do próprio diagnóstico. Amplamente utilizadas em clínica e em pesquisa, as escalas de avaliação são capazes de medir variáveis tanto da experiência interna quanto externamente observáveis. As escalas de avaliação da depressão mais conhecidas e utilizadas são: Inventário de Depressão de Beck, Avaliação Standard de Transtornos Depressivos (SADD), Escala Zung de Auto-avaliação para Depressão, Escala de Hamilton para Depressão, entre outras ${ }^{9-13}$.

Vários foram os estudos que buscaram verificar a ocorrência de depressão em pacientes com dor crônica, e foi possível comprovar que a maioria desses pacientes apresentavam sintomas depressivos ${ }^{8,14-19}$. Conclui-se que a depressão é extremamente comum em pacientes com dor crônica, mas a relação entre essas duas condições continua controversa ${ }^{16}$. Tanto a depressão como a dor crônica são formas comuns de somatização e, assim como a dor pode ser uma expressão somática do desconforto psicológico, a depressão pode ser uma resposta à dor $\mathrm{Crônica}^{20}$.

As mulheres atendidas em nosso ambulatório apresentam queixas emocionais que podem estar relacionadas a uma sintomatologia depressiva. A presença desta acarreta sofrimento psicológico e poderia dificultar o tratamento da endometriose, prejudicando principalmente a adesão da paciente ao próprio tratamento. Por isso, o estudo teve como objetivo avaliar a freqüência de depressão em mulheres portadoras de endometriose e com queixa atual de dor pélvica crônica, para determinar a necessidade ou não de atendimento psicológico de rotina.

\section{Métodos}

Dentreas pacientes atendidas no Ambulatório de Endometriose do Departamento de Tocoginecologia do Centro de Atenção Inte- gral à Saúde da Mulher (CAISM/FCM/ UNICAMP), durante o período de fevereiro de 2000 a janeiro de $200 \mathrm{I}$, foram avaliadas 50 pacientes atendidas consecutivamente e que se adequavam aos critérios estabelecidos para - estudo: diagnóstico confirmado de endometriose e presença de dor pélvica crônica há pelo menos seis meses.

As pacientes foram avaliadas pela Seção de Psicologia do Ambulatório de Endometriose no dia agendado para o retorno do atendimento médico, antes ou depois da consulta. $O$ atendimento psicológico faz parte da rotina do ambulatório e é oferecido a todas aspacientes.

A amostra foi avaliada por meio de instrumento padronizado e os dados coletados através de entrevista individual, realizada em ambiente privativo, estando presente somente a psicóloga e a paciente. No início da entrevista, a paciente era informada do objetivo do questionário, o procedimento que seria realizadoe o tratamento ético das informações. Não houve nenhuma negativa quanto à participação, nem mesmo desistência. A população foi caracterizada quanto à idade, estado civil, religião, número de filhos e uso de medicação.

Para investigar a prevalência da depressão nas pacientes selecionadas, utilizou-se o Inventário de Depressão de Beck ("Beck Depression Inventory"; BDI) ${ }^{21}$, validado em nosso meio ${ }^{22}$. Este inventário é um instrumento de auto-avaliação que permite discriminar a sintomatologia depressiva, amplamente utilizado tanto em pesquisa como em clínica.

Em 1996, Gorestein et al. ${ }^{22}$ confirmarama validade discriminante da versão em português do BDI, pela sua capacidade de diferenciar pacientes deprimidos de ansiosos e de sujeitos normais. Os itens do BDI, utilizados como critérios de avaliação neste estudo para verificar a presença de depressão numa população específica, preenchem os critérios estabelecidos pela Classificação de Transtornos Mentais e de Comportamentos do CID - I0 para o diagnóstico de depressão em pesquisa ${ }^{23}$.

$\mathrm{OBDl}$ é composto de $2 \mathrm{I}$ itens, incluindo sintomas e atitudes, cuja intensidade varia de 0 a 3. Os itens referem-se à tristeza, pessimismo, sensação de fracasso, falta de satisfação, sensação de culpa, sensação de punição, autodepreciação, auto-acusações, idéias suicidas, crises de choro, irritabilidade, retração social, indecisão, distorção da imagem corpo- 
ral, inibição para o trabalho, distúrbio do sono, fadiga, perda de apetite, perda de peso, preocupação somática e diminuição de libido ${ }^{22}$.

Os escores foram classificados de acordo com o critério de pontos de corte do "Center for Cognitive Therapy", que define: menor que $10=$ sem depressão ou depressão mínima, de $10 \mathrm{a} 18=$ depressão de leve a moderada, de 19 a $29=$ depressão moderada a grave, e de 30 a 63 = depressão grave ${ }^{22}$.

Após a coleta de dados, as informações foram inseridas em planilha do Microsoft Excel 97, com posterior análise descritiva dos resultados. Para correlacionar o grau de depressão com o uso de medicação hormonal foi utilizado análise comparativa e aplicado o teste Quiquadrado de Yates ${ }^{24}$.

\section{Resultados}

Aamostra avaliada foi composta de mulheres com diagnóstico de endometriose e dor pélvica crônica, com faixa etária entre 24 e 48 anos, sendo a média de 35,3 . Quanto ao estado civil, $86 \%$ relataram união e I $4 \%$ declararam estar sem parceiro. Quanto à religião, $64 \%$ católicas, $20 \%$ evangélicas, $8 \%$ sem credo e $8 \%$ outras religiões. Do total, $22 \%$ sem filhos, $52 \%$ um ou dois filhos, $26 \%$ três ou mais. Amaioria (66\%) estava utilizando medicação - análogo do GnRH, Acetato de Medroxiprogesterona de Depósito (AMP-D) ou pílula combinada - e $34 \%$ estavam sem medicação.

Foi possível identificar a presença de depressão em $92 \%$ das pacientes avaliadas. Em $36 \%$ das pacientes observou-se depressão leve, seguido de $34 \%$ de depressão moderada, $22 \%$ de depressão grave e $8 \%$ sem depressão ou depressão mínima. O escore médio de depressão detectado na amostra foi 20,8 , e a mediana, 20 . O escore máximo possível era de 63 pontos. Dos 50 escores obtidos pela avaliação, apenas uma apresentou-se igual a 0 , istoé, sem nenhuma alteração de comportamento. O escore mais elevado foi de $4 I$ pontos, onde $\mid 8$ dos $2 I$ itens do BDI foram assinalados entre intensidade de $I$ a 3 .

Encontrou-se entre as pacientes que estavam utilizando medicação hormonal (66\%), $31 \%$ destas apresentaram depressão, enquanto que nas pacientes que não relataram 0 uso de medicação hormonal (34\%), a presença de depressão ocorreu

\begin{tabular}{lccccc}
\hline & \multicolumn{4}{c}{ Tabela I - Grau de depressäo correlacionado ao uso de medicação } \\
\hline & \multicolumn{2}{c}{ com medicação } & & \multicolumn{2}{c}{ sem medicação } \\
\cline { 2 - 3 } \cline { 5 - 6 } sem depressão & $n$ & $\%$ & & $n$ & $\%$ \\
depressãoleve & 2 & 4 & & 6 & 12 \\
depressãomoderada & 12 & 24 & & 5 & 10 \\
depressãograve & 12 & 24 & & 4 & 8 \\
\hline
\end{tabular}

Diferenças não significativas para comparação de gurpos sem depressão e depressão leve comparado com depressão moderada egrave $(p=0.99)$

Figura I - Frequeência de alteraçōes dos itens do BDI na amostra avaliada

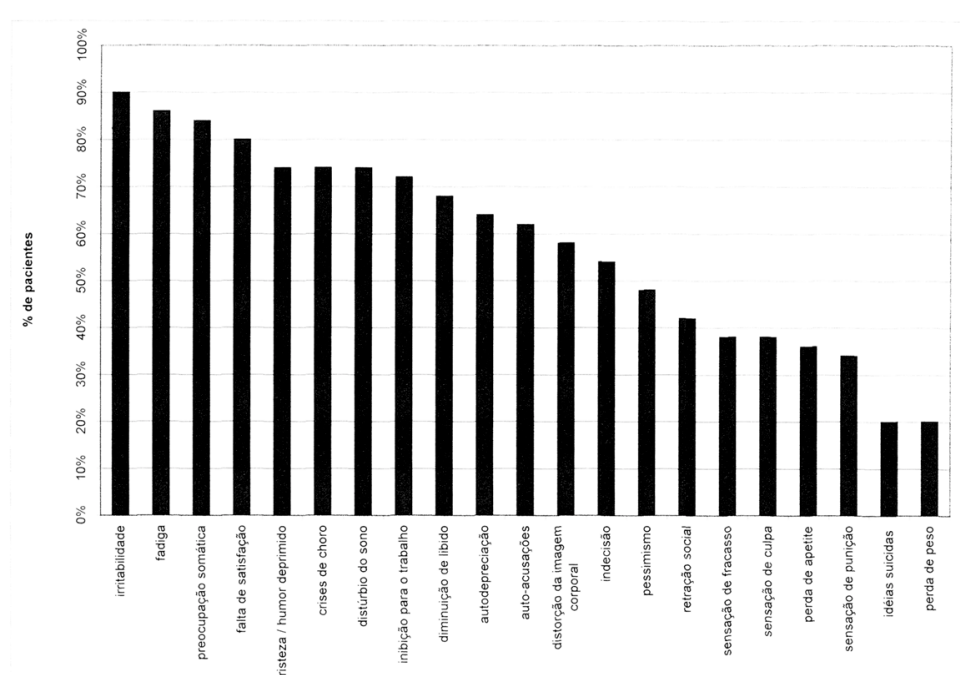

em $30 \%$ delas, diferenças não significativas. (Tabela I).

Foi possível identificar que dos $2 \mathrm{I}$ itens do BDI, I 3 foram pontuados (entre I a 3 de intensidade) por mais da metade da amostra. Destacam-se: irritabilidade (90\%), fadiga (86\%), preocupação somática (84\%), falta de satisfação (80\%), tristeza / humor deprimido (74\%), crises de choro (74\%), distúrbios do sono (74\%), inibição para o trabalho (72\%). Os menores foram: idéias suicidas (20\%) e perda de peso (20\%) (Figura I).

\section{Discussão}

No presente estudo, foi possível constatar a prevalência de depressão de intensidade variadas em $92 \%$ das mulheres entrevistadas. Não pudemos encontrar referências nacionais que correlacionam endometriose e grau de depressão. Em 1995, Waller etal..$^{25}$ realizaram um estudo comparativo entre mulheres come sem endometriose, sintomáticas ou não, utilizando o BDI. No grupo de mulheres com endometriose e dor pélvica crônica, a prevalência de depressão encontrada foi de $23,5 \%$. Vale ressaltar que foram avaliadas nesse grupo apenas 18 mulheres, porém a porcentagem foi bem menor do que a encontrada em nossa amostra.

A prevalência de depressão em pacientes com dor crônica em geral pode chegar a $78 \%$, segundo Pimenta ${ }^{8,15,17}$. As diferenças encontradas nos estudos que avaliam a associação entre depressão e dor crônica devem-se principalmente pela maneira como cada paciente utiliza sua estrutura cognitiva para adaptar-seà experiência dolorosa, apresentando ou não uma depressão ${ }^{8}$. É reconhecido que a relação entre essas duas condições ainda não foi claramente descrita, não possibilitando na maioria das ve- 
zes, identificar qual condição precede a outra.

Entendemos aqui que a presença da depressão nesse grupo de mulheres não decorre apenas do processo de adaptação e estresse relativos a endometriose e sintomas, mas também de características emocionais e cognitivas próprias de cada paciente. Sendo a doença e a dor condições crônicas, interferem significativamente na qualidade de vida dessas mulheres, ocasionando maior comprometimento de suas funções vitais, provocando alterações em suas percepções cognitivas, dificultando seriamente a adesão ao tratamento, gerando assim, resultados menos satisfatórios no controle da doençae de seus sintomas. $O$ quadro depressivo é facilmente instalado nestes casos devido ao comprometimento cognitivo e das funções vitais.

A sintomatologia da depressão é heterogênea, variando de indivíduo para indivíduo. No presente estudo, identificou-se através do $\mathrm{BDI}$, que mais de $80 \%$ das pacientes queixaram-se de irritabilidade, fadiga e preocupação somática. Dentre os 13 itens de aspectos cognitivos-afetivos que o instrumento permite avaliar, sete estavam alterados em mais de $50 \%$ da amostra (irritabilidade, falta de satisfação, tristeza, crises de choro, autodepreciação, auto-acusações e indecisão). Em contrapartida, dos oito itens que avaliam queixas somáticas e de desempenho, seis estavam alterados em mais de $50 \%$ da amostra, o que talvez fosse esperado, visto que as pacientes com dor crônica (de qualquer origem) sofrem uma queda significativa no desempenho físico. Os itens do BDI que avaliam o pessimismo, a retração social, sensação de fracasso e culpa, mostraram-se alterados em mais de $30 \%$ das pacientes. Idéias suicidas estiveram presentes em apenas $20 \%$. Resultado esse esperado, pois na maioria das vezes, idéias suicidas estão exacerbadas em pacientes com depressãograve.

A falta de correlação significativa entre a presença da depressão e uso da medicação foi um achado inesperado neste estudo, já que 0 uso destes medicamentos hormonais, em especiais os análogos do $\mathrm{GnRH}$, favoreceriam uma incidência maior de depressão, segundo relatos de alguns estudos ${ }^{26-28}$.

É possível afirmar, com base nos dados do presente estudo e com dados descritos anteriormente por Angelotti ${ }^{14}$, que as pacientes acometidas pela dor crônica reagem de forma exacerbada, produzindo efeitos nos comportamentos diários que prolongam-se ao passar do tempo, determinando algumas ações que as tornam impotentes e sem habilidades para controlar a intensidade de seu problema. Os fatores psicológicos que fazem parte da síndrome dolorosa, tais como a mudança no humor e a ansiedade, podem contribuir para melhorar ou piorar o estado atual da paciente. Os pensamentos negativos e autodestrutivos estão exacerbados e podem provocar efeitos profundos, generalizando os pensamentos distorcidos de forma inadequada.

Assim como os fatores biológicos, os fatores sociais, culturais e psicológicos são de importância fundamental na mediação do processamento da dor, pois embora seja subjetiva, a dor não é abstrata. É sentida por alguém que precisa ser respeitado e compreendido na sua realidade e totalidade para que possa ser realmente tratado ${ }^{29}$.

Com a aquisição de uma doença, muitos pacientes se deparam com a perda de um corpo saudável eativo, que pode significar para muitos, a perda da autonomia e da independência. Ocorrem também perdas significativas no círculo social em decorrência das limitações que a doença, e principalmente a dor, trazem para a pessoa, alterando a dinâmica social e afetiva de sua vida. Podem ocorrer perdas de emprego e da estabilidade econômica, e principalmente das relações afetivas. Estas, por si só geradoras de insegurança e ansiedade, são agravadas pela situação de dependência que muitos pacientes que sofrem de dor crônica assumem. Dessa forma, a doença ocupa um lugar central na vida da paciente, alterando não só seu desempenho social e econômico, mas principalmente suas percepções cognitivas, resultando muitas vezes em uma depressão. Por esse motivo, os avanços das ciências da saúde contribuíram para propagar a necessidade da assistência integral, a partir de uma abordagem multifatorial para a compreensão da etiologia da enfermidade, aprimorando técnicas e terapêuticas, para possibilitar a recuperação integral da paciente ${ }^{30}$.

Oobjetivo da psicologia voltada para oatendimento hospitalar e ambulatorial é resgatar a qualidade devida do pacienteque foi interrompida com a doença, buscando compreender 0 acontecer psíquico neste dado momento. Visa compreender os mecanismos psíquicos de determinada patologia, sua função na vida do paciente, para tornar possível uma visão ampla do que está acontecendo, auxiliando a equipe e o paciente no processo terapêutico ${ }^{30}$.
Em nossa experiência clínica, o atendimento multiprofissional tem demonstrado ser de grande importância no tratamento e seguimento de mulheres com endometriose, onde acreditamos que, buscando uma visão integrada das ciências, resgata-se a visão global do ser humano e suas apresentações como resultantes de um conjunto biopsicosocial.

\section{ConClusão}

Concluímos, com os dados obtidos, que a grande maioria das pacientes investigadas necessitam de atendimento psicológico devido a alta prevalência de depressão, focalizando aspectos psíquicos envolvidos no processo de adoecimento e depressivo. Nestes casos, a depressão deve ser também tratada e não apenas entendida como resultado esperado do sofrimento decorrente da cronicidade do sintoma doloroso.

Acreditamos ainda na necessidade de novos estudos para verificar a associação dos aspectos psicológicos no desenvolvimento da endometriose edos sintomas relacionados aessa doença.

\section{SUMMARY}

EVALUATION OF THE FREQUENCY OF DEPRESSION IN PATIENTS WITH ENDOMETRIOSIS AND PELVIC PAIN

OBJECTIVE. Evaluate the frequency ofdepression in women with endometriosis and actual symptom of pelvic pain to determine the necessity of psychological support.

METHODS. A total of 50 womenaged 24 to 48 withendometriosis and referringpelvicpain. The prevalence of depression was evaluated by Beck's Depression Inventory adapted to the Brazilian population.

RESULTS. It was found that $92 \%$ of the subjects presented depressive symptoms, being $56 \%$ from moderate to high levels. Although $66 \%$ of the women were using hormonal medication to treat endometriosis, there was no significant correlation between the presence of depression and the use of these medications.

CONCLUSION. Theresultsshowedahighprevalence of depression symptoms in these patients confirming thenecessity of psychologicalsupport. [Rev Assoc Med Bras 2002; 48(3): 217-2I]

KeY Words: Endometriosis. Pelvic pain. Depression. Psychological care. 


\section{REFERÊNCIAS}

I. Abrão MS, Abrão CM, Reiss RW, Vasconcelos E. Ansiedade, estresse e endometriose. In: Abrão MS. Endometriose: uma visão contemporânea. Rio de Janeiro: Revinter; 2000. p.249-57.

2. Barbosa CP, Cordts EB. Endometriomas ovarianos. Femina 1998; 26:805-10.

3. Camargos AF, Lemos CNCD. Endometriose. In: HALBE HW. Tratado de ginecologia 3a ed. São Paulo: Ed. Roca; 2000. v.2, p. I324-34.

4. Pineda RL. Diagnostico de la endometriosis. In: Acosta AA, Warman S. Endometriosis y esterilidad. Rio de Janeiro: Roussel Udaf; 1998. p.2I-32.

5. Low WY, Edelmann RJ, Sutton C. A psychological profile of endometriosis patients in comparison to patients with pelvic pain of other origins. J Psychosom Res 1993; 37: I I I-7.

6. Gruppo Italiano per lo Studio dell'Endometriosi. Relationship between stage, site and morphological characteristics of pelvic endometriosis and pain. Hum Reprod 200I; 16:2668-7I.

7. Almeida FM. A prática invasiva na dor pélvica. Femina 200I; 29:149-50.

8. Pimenta CAM. Fundamentos teóricos da dore de sua avaliação. In: Carvalho MMMJ, organizador. Dor: um estudo multidisciplinar. São Paulo: Ed. Summus; 1999. p.31-46.

9. DSM-IV. Manual diagnóstico e estatístico de transtornos mentais. 4a ed. Porto Alegre: Artes Médicas; 2000.

10. Hetem LAB. Diagnóstico, classificação (CIDI0) e diagnóstico diferencial dos transtornos depressivos. Arq Bras Med 1994; 68:163-7.

II. Organização Mundial da Saúde. Classificação de transtornos mentais e de comportamentos da CID-10: Descrições clínicas e diretrizes diagnosticas. Porto Alegre: Artes Médicas; 1993.

12. Kaplan HI, Sadock BJ, GrebbJA. Compêndio de psiquiatria clínica: ciências do comportamento e psiquiatria clínica. 7a ed. Porto Alegre: Artes Médicas; 1997.

13. Calil HM, Pires MLN. Aspectos gerais das escalas de avaliação de depressão. Rev Psiquiatr Clin 1 998; 25:240-4.

14. Angelotti G. Dor crônica: aspectos biológicos, psicológicos e sociais. In: Camon, VAA, organizador. Psicossomática e a psicologia da dor. São Paulo: Ed. Pioneira; 200 I. p. I I 3-29.

15. Pimenta CAM, Koizumi MS, Teixeira MJ. Dor, depressão e conceitos culturais. Arq Neuropsiquiatr 1997; 55:370-80.

16. FigueiróJAB. Aspectospsicológicosepsiquiátricos da experiência dolorosa. In: Carvalho MMMJ, organizador. Dor: um estudo multidisciplinar. São Paulo: Ed. Summus; 1999. p. I40-58.

17. Pimenta CAM, Koizumi MS, Teixeira MJ. Dor crônica e depressão: estudo em 92 doentes. Rev Esc Enferm USP 2000; 34:76-83.

I8. Wade JB, Dougherty LM, Hart RP, Cook DB. Patterns of normal personality structure among chronic pain patients. Pain 1992; 48:37-43.

19. Doan BD, Wadden NP. Relationships between depressive symptoms and descriptions of chronic pain. Pain 1989; 36:75-84.

20. Ford CV. The somatizing disorders. Psychosomatics 1986; 27:327-37.

2I. BeckAT, Rush AJ, Shaw BF, Emery G. Terapia cognitiva da depressão. Porto Alegre: Artes Médicas; 1997. p.29I-2.

22. Gorestein C, Andrade L. Inventário de depressão de Beck: propriedades psicomé- tricas da versão em português. Rev Psiquiatr Clín 1998; 25:245-50.

23. Organização Mundial de Saúde. Classificação de transtornos mentais e de comportamentos da CID-10: Critérios diagnósticos para pesquisa. Porto Alegre: Artes Médicas; 1998.

24. Armitage P. Statistical methods in medical research. New York: John Wiley and Sons; 1974.

25. Waller KG, Shaw RW. Endometriosis, pelvic pain, and psychological functioning. Fertil Steril 1995; 63:796-800.

26. Steingold KA, Cedars M, Lu JK, Randle D, Judd $H L$, Meldrum DR. Treatment of endometriosis with a long-acting gonadotropin-releasing hormone agonist. Obstet Gynecol I 987; 69:403-11.

27. Warnock JK, Bundren JC, Morris DW. Sertraline in the treatment of depression associated with gonadotropin-releasing hormone agonist therapy. Biol Psychol 1998; 43:464-5.

28. Bernik MA, Vieira Filho AHG. Depressão na mulher. Rev Bras Med 1998; 55:77I-80.

29. Carvalho MMMJ. Palavras iniciais. In: Carvalho MMMJ, organizador. Dor: um estudo multidisciplinar. São Paulo: Ed. Summus; 1999. p. 19-21.

30. Santos CT, Sebastiani RW. Acompanhamento psicológico à pessoa portadora de doença crônica. In: Camon VAA, organizador. E a psicologia entrou no hospital... São Paulo: Ed. Pioneira; 1996. p. 147-75.

Artigo recebido: |4/08/200|

Aceito para publicação: 30/01/2002

\section{OPINIÃO DO LEITOR}

A nova Ramb abre espaço para o leitor na seção "Correspondências". Envie sua opinião ou sugestão sobre a revista ou artigos de seu interesse. Participe também enviando suas dúvidas ou sugestões sobre condutas práticas para a seção "À beira do leito".

Sua colaboração é muito importante para aprimorarmos o nosso veículo de comunicação. Rua São Carlos do Pinhal, 324 Cep: 01333-903 - São Paulo - SP - E-mail:ramb@amb.org.br 